\title{
Analysis of a Separable STAP Algorithm for Very Large Arrays
}

\author{
Jie Chen, Feng Jiang and A. Lee Swindlehurst \\ Center for Pervasive Communications and Computing (CPCC) \\ Department of EECS, University of California, Irvine \\ Irvine, CA 92697, USA \\ \{jie.chen, feng.jiang, swindle\}@uci.edu
}

\begin{abstract}
Studies of massive MIMO in wireless communications have recently attracted significant attention. Here we study the benefits of very large arrays in space-time adaptive processing (STAP) for radar by analyzing the performance of a reduced-dimension separable STAP algorithm that exploits the large-array assumption. In particular, we begin by studying the behavior of the algorithm for clairvoyant interference covariance matrices with orthogonality assumptions on the steering vectors, and show that in the asymptotic sense this simplified scheme performs as well as the fully adaptive STAP method. We then appeal to random matrix theory to analyze performance when the covariance matrix is estimated using secondary data.
\end{abstract}

\section{INTRODUCTION}

Recently, considerable attention has been focused on "massive" multiple-input multiple-output (MIMO) systems in wireless communications, in which base stations are equipped with a very large number of antennas in order to dramatically improve system capacity. The resulting capacity gains can often be achieved with relatively simple signal processing, due to the asymptotic orthogonality of the wireless channels. Hence massive MIMO is regarded as a promising technology for the next generation of wireless communication systems. Correspondingly, the benefits of very large arrays can also be exploited in other areas as well, such as space-time adaptive processing (STAP) [1]. In this paper, we analyze the performance of a particular reduced-dimension separable STAP algorithm for large arrays taking the effect of finite secondary data support into account. We study the performance of the algorithm for clairvoyant interference covariance matrices with orthogonality assumptions on the steering vectors, and show that in the asymptotic sense this scheme performs as well as the fully adaptive STAP method. Then we use random matrix theory to analyze the signal-to-interference-plus-noise ratio (SINR) as a function of the number of training samples when the covariance matrix is estimated using secondary data.

So-called "fully adaptive" STAP methods [2] have very high complexity when large arrays are employed, and thus one often resorts to reduced-dimension or reduced-rank algorithms [1]-[4] in order to achieve a reasonable trade-off between performance and complexity. Many other reduced complexity approaches have been proposed over the years, including the eigencanceler [5], [6], iterative methods such as [7], the multistage Wiener filter [8], [9], and parametric clutter modeling [10]. Here we focus on a simple method that exploits the large array assumption to separate the spatial and temporal interference cancellation, and analyze its performance using random matrix theory [11], which has been found to be a useful tool in the analysis of very large wireless communication systems. Recently, it has also been applied to the study of STAP algorithms and other sample covariance matrix problems [12]. For example, the authors of [13] studied STAP in amplitude heterogeneous clutter environments, where the power of the interference in the secondary data is different from that in the range bin of detection. A closed-form expression of the SINR loss was given, and the asymptotic performance was clearly established using random matrix theory. In [14], the authors studied the asymptotic SINR loss of knowledge-aided STAP algorithms for cases involving either accurate or inaccurate a priori knowledge.

\section{System ModeL}

The system model is depicted in Fig. 1, in which a platform is moving parallel to the $x$-axis and a target is located anywhere in space and moving in an arbitrary direction. Angles $\theta$ and $\phi$ respectively represent the elevation and azimuth angles of the target relative to the airborne platform. The platform is assumed to be equipped with an antenna array of $N$ elements, and the transmitter on the platform is assumed to emit $M$ pulses per coherent processing interval (CPI). For a given range gate, the received data at pulse $m$ is denoted by $\mathbf{x}_{m}$. Defining $\chi=\left[\begin{array}{lll}\mathbf{x}_{1}^{T} & \ldots & \mathbf{x}_{M}^{T}\end{array}\right]^{T}$, the STAP detection problem is defined by the two hypotheses

$$
\left\{\begin{array}{l}
H_{0}: \chi=\chi_{c}+\chi_{n} \\
H_{1}: \chi=\chi_{t}+\chi_{c}+\chi_{n}
\end{array},\right.
$$

where $\chi_{t}$ is the signal due to the target, $\chi_{c}$ is due to clutter, and $\chi_{n}$ represents other noise and interference. The terms $\chi_{c}$ and $\chi_{n}$ are assumed to be random vectors with covariance matrices $\mathbf{R}_{c}$ and $\mathbf{R}_{n}$, respectively.

The detection statistic for fully adaptive STAP is $z=\mathbf{w}^{H} \chi$, where $\mathbf{w}=\mathbf{R}^{-1} \mathbf{v}_{t}$ is the weighting vector, $\mathbf{R}$ is the sum of $\mathbf{R}_{c}$ and $\mathbf{R}_{n}$, and $\mathbf{v}_{t}$ is the target steering vector. The post-processing SINR at the platform determines the overall detection performance. From [1], the general SINR expression is

$$
S I N R=\frac{\sigma^{2} \xi_{t}\left|\mathbf{w}^{H} \mathbf{v}_{t}\right|^{2}}{\mathbf{w}^{H} \mathbf{R} \mathbf{w}},
$$




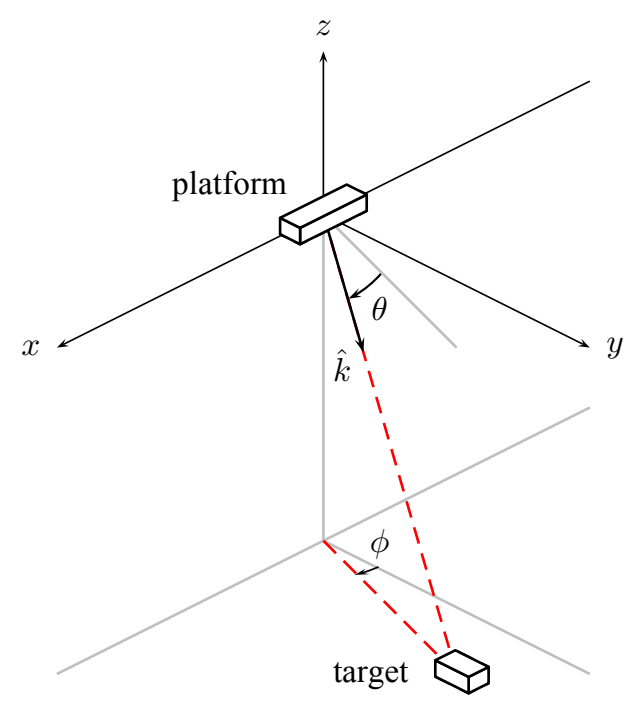

Fig. 1: System model

and the SINR of the fully adaptive STAP method is

$$
S I N R=\sigma^{2} \xi_{t} \mathbf{v}_{t}^{H} \mathbf{R}^{-1} \mathbf{v}_{t},
$$

where $\sigma^{2}$ is the noise power per element, and $\xi_{t}$ is the singlepulse signal-to-noise ratio (SNR) for a single antenna element at the receive side.

The fully adaptive STAP algorithm is often prohibitively costly to implement since the dimensionality of $\mathbf{R}$ is $M N$, which makes calculation of matrix inverse difficult even for a system with a moderate number of antennas. In this paper, we consider the performance of a simple separable STAP algorithm that effectively exploits the benefits of very large antenna arrays. In this approach, the received data are first processed by a spatial-only non-adaptive beamformer $\mathbf{f}$, followed by an adaptive filter in the temporal domain. Processing with the spatial-only filter leads to the transformed data vector

$$
\tilde{\chi}=(\mathbf{I} \otimes \mathbf{f})^{H} \chi,
$$

where $\mathbf{I}$ is the identity matrix, and $\otimes$ denotes the Kronecker product. Letting $\mathbf{F}=\mathbf{I} \otimes \mathbf{f}$, the transformed covariance matrix is

$$
\tilde{\mathbf{R}}=\mathbf{F}^{H} \mathbf{R F} .
$$

Similarly, the transformed target steering vector is $\tilde{\mathbf{v}}_{t}=\mathbf{F}^{H} \mathbf{v}_{t}$. For simplicity we assume $\mathbf{f}$ is a normalized vector, i.e., $\|\mathbf{f}\|=$ 1 , and thus it is obvious that $\mathbf{F}^{H} \mathbf{F}=(\mathbf{I} \otimes \mathbf{f})^{H}(\mathbf{I} \otimes \mathbf{f})=\mathbf{I}$.

For the reduced-dimension algorithm, the detection statistic is

$$
\tilde{z}=\tilde{\mathbf{w}}^{H} \tilde{\chi}
$$

where $\tilde{\mathbf{w}}=\tilde{\mathbf{R}}^{-1} \tilde{\mathbf{v}}_{t}=\left(\mathbf{F}^{H} \mathbf{R F}\right)^{-1} \mathbf{F}^{H} \mathbf{v}_{t}$. The SINR of this algorithm can be calculated from (2) and is

$$
S I N R=\sigma^{2} \xi_{t} \mathbf{v}_{t}^{H} \mathbf{F}\left(\mathbf{F}^{H} \mathbf{R F}\right)^{-1} \mathbf{F}^{H} \mathbf{v}_{t} .
$$

In practice, matrix $\mathbf{R}$ can only be estimated using secondary data. Letting $\hat{\mathbf{R}}$ denote the estimate, the weighting vector is

$$
\mathbf{w}=\mathbf{F}\left(\mathbf{F}^{H} \hat{\mathbf{R}} \mathbf{F}\right)^{-1} \mathbf{F}^{H} \mathbf{v}_{t},
$$

and from (2) we know the corresponding SINR is given by

$$
S I N R=\frac{\sigma^{2} \xi_{t}\left|\mathbf{v}_{t}^{H} \mathbf{F}\left(\mathbf{F}^{H} \hat{\mathbf{R}} \mathbf{F}\right)^{-1} \mathbf{F}^{H} \mathbf{v}_{t}\right|^{2}}{\mathbf{v}_{t}^{H} \mathbf{F}\left(\mathbf{F}^{H} \hat{\mathbf{R}} \mathbf{F}\right)^{-1} \mathbf{F}^{H} \mathbf{R} \mathbf{F}\left(\mathbf{F}^{H} \hat{\mathbf{R}} \mathbf{F}\right)^{-1} \mathbf{F}^{H} \mathbf{v}_{t}} .
$$

The most frequently used method to estimate $\mathbf{R}$ is sample matrix inversion (SMI). The estimated covariance matrix is $\hat{\mathbf{R}}=\frac{1}{K} \mathbf{X X}^{H}$ and $\mathbf{X}=\left[\chi_{1}, \ldots, \chi_{K}\right]$ contains training samples from $K$ different range intervals. We will assume the sample covariance matrix is used to estimate $\mathbf{R}$ in what follows.

\section{VERY LARGE ARRAY ANALYSis FOR 2D ARRAYS}

In this section, we analyze the performance of the reduceddimension algorithm for very large planar arrays, and compare it to the fully adaptive STAP algorithm. We can expect that an enormous amount of antenna elements in a two-dimensional array configuration results in extremely narrow beams, which leads to very high discrimination of the target from the clutter and interference as shown in Fig. 2; in essence, as long as the target is elevated above the ground, its steering vector will be asymptotically orthogonal to any clutter patch in the same range bin. Hence this system can separate the target from nearby clutter patches even when they are very close in the azimuth or elevation domain. As a result, we will show that the performance of the simple separable algorithm is asymptotically equal to that of the fully adaptive algorithm. A closed-form expression for the asymptotic SINR is also given at the end of the section, which can be used to quickly estimate the required number of antenna elements for a given performance target in STAP system designs.

\section{A. Rectangular Array Model}

We assume that a $P$ by $Q$ rectangular array is used, and the notation used here is similar to that adopted in [15], [16]. The target spatial frequency is $\vartheta_{t}=p \vartheta_{x}+q \vartheta_{z}$, where $\vartheta_{x}=$ $d_{x} / \lambda_{0} \cos \theta_{t} \sin \phi_{t}$ and $\vartheta_{z}=d_{z} / \lambda_{0} \sin \theta_{t}$. The target Doppler frequency is $\varpi_{t}=2 v_{t} f_{r} / \lambda_{0}$, and the target steering vector is

$$
\mathbf{v}_{t}\left(\varpi_{t}, \vartheta_{x}, \vartheta_{z}\right)=\mathbf{b}\left(\varpi_{t}\right) \otimes \mathbf{a}\left(\vartheta_{x}\right) \otimes \mathbf{e}\left(\vartheta_{z}\right)
$$

where

$\mathbf{b}=\left[\begin{array}{c}1 \\ e^{j 2 \pi \varpi_{t}} \\ \vdots \\ e^{j(M-1) 2 \pi \varpi_{t}}\end{array}\right], \mathbf{a}=\left[\begin{array}{c}1 \\ e^{j 2 \pi \vartheta_{x}} \\ \vdots \\ e^{j(P-1) 2 \pi \vartheta_{x}}\end{array}\right], \mathbf{e}=\left[\begin{array}{c}1 \\ e^{j 2 \pi \vartheta_{z}} \\ \vdots \\ e^{j(Q-1) 2 \pi \vartheta_{z}}\end{array}\right]$.

The target signal, $\chi_{t}$, is equal to $\alpha_{t} \mathbf{v}_{t}\left(\varpi_{t}, \vartheta_{x}, \vartheta_{z}\right)$, where $\alpha_{t}$ is the complex amplitude. 


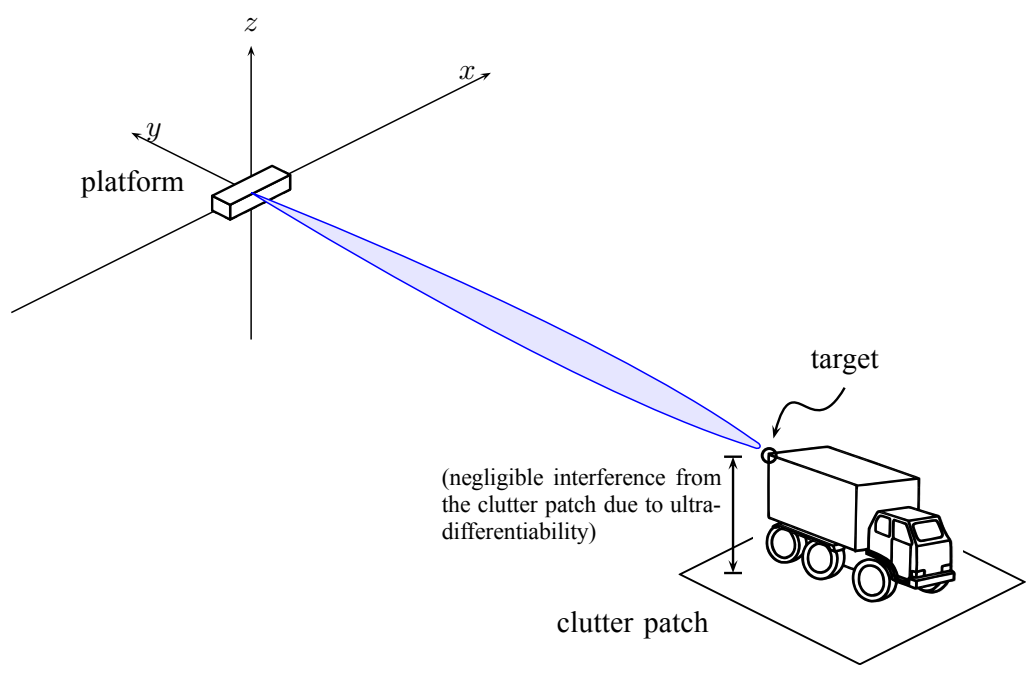

Fig. 2: Target is moderately above the ground clutter patch

The overall steering vector can also be written as $\mathbf{v}\left(\varpi, \vartheta_{x}, \vartheta_{z}\right)=\boldsymbol{\beta}(\varpi) \otimes \boldsymbol{\alpha}(\vartheta)$, where $\boldsymbol{\beta}=\mathbf{b}(\varpi)$, and $\boldsymbol{\alpha}=\mathbf{a}\left(\vartheta_{x}\right) \otimes \mathbf{e}\left(\vartheta_{z}\right)$. The normalized spatial filtering vector is then $\mathbf{f}=\frac{1}{\sqrt{P Q}} \boldsymbol{\alpha}$.

\section{B. Orthogonality Assumption}

The analysis in this section relies on the fact that as the number of antenna elements goes to infinity, the produced beams towards different spatial directions are asymptotically orthogonal. Let $\hat{\boldsymbol{\beta}}=\frac{1}{\sqrt{M}} \boldsymbol{\beta}, \hat{\boldsymbol{\alpha}}=\frac{1}{\sqrt{N}} \boldsymbol{\alpha}$ and $\hat{\mathbf{v}}=\frac{1}{\sqrt{M N}} \mathbf{v}$ be the normalized versions of $\boldsymbol{\beta}, \boldsymbol{\alpha}$ and $\mathbf{v}$, respectively. For an arbitrary steering vector pair $\hat{\mathbf{v}}_{i}$ and $\hat{\mathbf{v}}_{j}, i \neq j$, the inner product of the two vectors is equal to

$$
\left(\mathbf{b}^{H}\left(\varpi_{j}\right) \mathbf{b}\left(\varpi_{i}\right)\right) \otimes\left(\mathbf{a}^{H}\left(\vartheta_{x j}\right) \mathbf{a}\left(\vartheta_{x i}\right)\right) \otimes\left(\mathbf{e}^{H}\left(\vartheta_{z j}\right) \mathbf{e}\left(\vartheta_{z i}\right)\right),
$$

and the inner products of the corresponding normalized spatial steering vectors are

$$
\left\{\begin{array}{l}
\hat{\mathbf{a}}^{H}\left(\vartheta_{j}\right) \hat{\mathbf{a}}\left(\vartheta_{i}\right)=\frac{1}{N} \sum_{n=1}^{N} e^{j(n-1) 2 \pi\left(\vartheta_{x i}-\vartheta_{x j}\right)} \\
\hat{\mathbf{e}}^{H}\left(\vartheta_{j}\right) \hat{\mathbf{e}}\left(\vartheta_{i}\right)=\frac{1}{N} \sum_{n=1}^{N} e^{j(n-1) 2 \pi\left(\vartheta_{z i}-\vartheta_{z j}\right)} .
\end{array}\right.
$$

It is easy to show that $\lim _{N \rightarrow \infty} \frac{1}{N} \sum_{n=0}^{N-1} e^{j n \theta}$ is non-zero only when $\theta=0$. In other words, the two spatial steering vectors are non-orthogonal only when they correspond to the same angle. Due to the structure of the full inner products in (11), the same claim applies to the full steering vector case, namely that $\mathbf{v}_{i}$ and $\mathbf{v}_{j}$ are orthogonal as long as their angles do not coincide, and this orthogonality is independent of their temporal relationship.

\section{SINR Performance Analysis}

The signal reflected from the target is in general contaminated by clutter. We will analyze the SINR performance of the reduced-dimension STAP algorithm under the orthogonality assumption discussed above. Without loss of generality, we assume there is no ranging ambiguity. Thus the clutter interference covariance matrix is

$$
\mathbf{R}_{c}=\sigma^{2} \sum_{i=1}^{N_{c}} \xi_{i} \mathbf{v}_{i} \mathbf{v}_{i}^{H}=\sigma^{2} M N \sum_{i=1}^{N_{c}} \xi_{i} \hat{\mathbf{v}}_{i} \hat{\mathbf{v}}_{i}^{H},
$$

where $N_{c}$ is the total number of clutter patches. The noise covariance matrix is assumed to be $\mathbf{R}_{n}=\sigma^{2} \mathbf{I}$, so we have

$$
\mathbf{R}=\mathbf{R}_{c}+\mathbf{R}_{n}=\sigma^{2} M N \sum_{i=1}^{N_{c}} \xi_{i} \hat{\mathbf{v}}_{i} \hat{\mathbf{v}}_{i}^{H}+\sigma^{2} \mathbf{I} .
$$

We want to find an analytic expression for (7) when the number of antenna elements is infinity. The calculation of the SINR in (7), $\mathbf{v}_{t}^{H} \mathbf{F}\left(\mathbf{F}^{H} \mathbf{R F}\right)^{-1} \mathbf{F}^{H} \mathbf{v}_{t}$, can be found by computing the product of some components including $\left(\mathbf{F}^{H} \mathbf{R F}\right)^{-1}$, $\mathbf{R}^{\frac{1}{2}} \mathbf{F}$ and $\mathbf{R}^{-\frac{1}{2}} \mathbf{v}_{t}$.

From the discussion on orthogonality in the previous subsection, we note that $\operatorname{span}\left\{\hat{\mathbf{v}}_{1}, \ldots, \hat{\mathbf{v}}_{N_{c}}\right\}$ forms an $N_{c^{-}}$ dimensional subspace of $\mathbb{C}^{M N}$, where $N_{c} \ll M N$. So we can find other orthonromal vectors $\hat{\mathbf{u}}_{i}, i=1, \ldots, M N-N_{c}$ in $\mathbb{C}^{M N}$ which together with $\hat{\mathbf{v}}_{i}, i=1, \ldots, N_{c}$ form an orthonormal basis of $\mathbb{C}^{M N}$. With the help of these vectors, the covariance matrix in (13) can be further eigen-decomposed as

$$
\mathbf{R}=\sum_{i=1}^{N_{c}} \sigma^{2}\left(M N \xi_{i}+1\right) \hat{\mathbf{v}}_{i} \hat{\mathbf{v}}_{i}^{H}+\sigma^{2} \sum_{i=1}^{M N-N_{c}} \hat{\mathbf{u}}_{i} \hat{\mathbf{u}}_{i}^{H} .
$$

Hence the square root of $\mathbf{R}$ is

$$
\mathbf{R}^{\frac{1}{2}}=\sum_{i=1}^{N_{c}}\left(\left(\sigma^{2}\left(M N \xi_{i}+1\right)\right)^{\frac{1}{2}}-\sigma\right) \hat{\mathbf{v}}_{i} \hat{\mathbf{v}}_{i}^{H}+\sigma \mathbf{I}
$$

and clearly

$$
\mathbf{R}^{-\frac{1}{2}}=\sum_{i=1}^{N_{c}}\left(\left(\sigma^{2}\left(M N \xi_{i}+1\right)\right)^{-\frac{1}{2}}-\frac{1}{\sigma}\right) \hat{\mathbf{v}}_{i} \hat{\mathbf{v}}_{i}^{H}+\frac{1}{\sigma} \mathbf{I} .
$$


Now we are ready to calculate $\left(\mathbf{F}^{H} \mathbf{R F}\right)^{-1}, \mathbf{R}^{\frac{1}{2}} \mathbf{F}$, and $\mathbf{R}^{-\frac{1}{2}} \mathbf{v}_{t}$, respectively. By utilizing the fact that $\hat{\mathbf{v}}_{i}, i=$ $1, \ldots, N_{c}$ and $\hat{\mathbf{u}}_{i}, i=1, \ldots, M N-N_{c}$ form an orthonormal basis, it is straightforward to see $\mathbf{R}^{\frac{1}{2}} \mathbf{F}=\sigma \mathbf{F}$. Similarly, we also have $\mathbf{R}^{-\frac{1}{2}} \hat{\mathbf{v}}_{t}=\frac{1}{\sigma} \hat{\mathbf{v}}_{t}$ and $\left(\mathbf{F}^{H} \mathbf{R F}\right)^{-1}=\frac{1}{\sigma^{2}} \mathbf{I}$. Putting everything together, we have the following analytic expression for the asymptotic SINR:

$$
S I N R=\sigma^{2} \xi_{t} \mathbf{v}_{t}^{H} \mathbf{F}\left(\mathbf{F}^{H} \mathbf{R F}\right)^{-1} \mathbf{F}^{H} \mathbf{v}_{t}=M N \xi_{t} .
$$

For purpose of comparison, we also see that the asymptotic SINR of the fully adaptive STAP is given by

$$
S I N R=\sigma^{2} \xi_{t} \mathbf{v}_{t}^{H} \mathbf{R}^{-1} \mathbf{v}_{t}=M N \xi_{t} .
$$

It is clear that the SINR of the proposed separable STAP algorithm is exactly the same as that of the fully adaptive algorithm when the number of antenna elements is infinity, but the separable scheme has a much lower computational complexity, since only an $M \times M$ rather than an $M N \times M N$ matrix must be inverted.

\section{Secondary SAmple Size Analysis}

The number of secondary data samples used to estimate $\mathbf{R}$ has a great impact on the performance of the system. In this section we study the SINR scaling law as a function of the secondary data support. As part of this analysis, we propose the following new theorem based on random matrix theory.

Theorem 1. Let $\mathbf{X}=\left[\begin{array}{lll}\mathbf{x}_{1} & \ldots & \mathbf{x}_{K}\end{array}\right]$, where for all $k \mathbf{x}_{k}$ is a zero-mean complex Gaussian random vector with covariance R. Vectors $\mathbf{x}_{i}$ and $\mathbf{x}_{j}$ are independent when $i \neq j$. Define a random matrix $\mathbf{A}=\frac{1}{K} \mathbf{F}^{H} \mathbf{X X}^{H} \mathbf{F}$, where $\mathbf{F} \in \mathbb{C}^{L \times M}$, $\mathbf{X} \in \mathbb{C}^{L \times K}$, and $M$ is a fixed number. As $K, L \rightarrow \infty$, we have

$$
\left|\mathbf{a}^{H}(\mathbf{A}-z \mathbf{I})^{-1} \mathbf{a}-\mathbf{a}^{H}(x(z) \mathbf{P}-z \mathbf{I})^{-1} \mathbf{a}\right| \longrightarrow 0,
$$

where $\mathbf{a} \in \mathbb{C}^{M \times 1}$ is an arbitrary fixed vector, $\mathbf{P}=\mathbf{F}^{H} \mathbf{R F}$, $x(z)=1-\frac{M}{K}-\frac{M}{K} z m_{F_{\mathbf{A}}^{K}}(z)$, and $m_{F_{\mathbf{A}}^{K}}(z)$ is the Stieltjes transform of $\mathbf{A}$.

This theorem is similar to one in [17]. The key difference is that in our case the dimensionality of $\mathbf{A}$ is always limited, and thus a new proof is required and will be provided in [18].

Let $K$ be the number of secondary data samples and $L=$ $M N$. Applying Theorem 1 to (9), we have that when $K$ is sufficiently large, the SINR is approximately equal to

$$
\frac{\sigma^{2} \xi_{t}}{1-x^{\prime}(0)} \mathbf{v}_{t}^{H} \mathbf{F}\left(\mathbf{F}^{H} \mathbf{R F}\right)^{-1} \mathbf{F}^{H} \mathbf{v}_{t}
$$

where $x^{\prime}(0)=-\frac{1}{K}\left\{\sum_{m=1}^{M} \frac{1}{\lambda_{m}\left(1-\frac{M}{K}\right)}\right\}$, and $\lambda_{m}$ are the eigenvalues of $\mathbf{F}^{H} \mathbf{R F}$.

The detailed derivation goes as follows. We want to evaluate the numerator and denominator of (9) separately. Define

$$
\left\{\begin{array}{l}
g(z)=\mathbf{v}_{t}^{H} \mathbf{F}\left(\mathbf{F}^{H} \hat{\mathbf{R}} \mathbf{F}-z \mathbf{I}\right)^{-1} \mathbf{F}^{H} \mathbf{v}_{t} \\
\gamma(z)=\mathbf{v}_{t}^{H} \mathbf{F}\left(x(z) \mathbf{F}^{H} \mathbf{R} \mathbf{F}-z \mathbf{I}\right)^{-1} \mathbf{F}^{H} \mathbf{v}_{t}
\end{array} .\right.
$$

TABLE I: System Parameters

\begin{tabular}{|c|c|}
\hline SNR for a single antenna element $\xi_{t}$ & $1 \mathrm{~dB}$ \\
\hline reflectivity $\gamma$ & $-3 \mathrm{~dB}$ \\
\hline noise power per element $\sigma^{2}$ & 1 \\
\hline range & $6000 \mathrm{~m}$ \\
\hline number of clutter patches & 360 \\
\hline pulse repetition frequency (PRF) $f_{r}$ & $300 \mathrm{~Hz}$ \\
\hline interelement spacing & $\lambda / 2$ \\
\hline frequency & $0.45 \mathrm{GHz}$ \\
\hline platform altitude & $3000 \mathrm{~m}$ \\
\hline platform velocity & $50 \mathrm{~m} / \mathrm{s}$ \\
\hline
\end{tabular}

Then the numerator of (9) is equal to $\left.\sigma^{2} \xi_{t} g^{2}(z)\right|_{z=0}$. Based on Theorem 1 we know $|g(z)-\gamma(z)| \rightarrow 0$, so asymptotically the numerator approaches

$$
\frac{1}{x(0)} \mathbf{v}_{t}^{H} \mathbf{F}\left(\mathbf{F}^{H} \mathbf{R F}\right)^{-1} \mathbf{F}^{H} \mathbf{v}_{t}
$$

The next step is to calculate the denominator. Define

$$
\left\{\begin{array}{l}
\mathbf{T}=\mathbf{F}\left(\mathbf{F}^{H} \mathbf{R F}\right)^{-\frac{1}{2}} \\
h(z)=\mathbf{v}_{t}^{H} \mathbf{T}\left(\mathbf{T}^{H} \hat{\mathbf{R}} \mathbf{T}-z \mathbf{I}\right)^{-1} \mathbf{T}^{H} \mathbf{v}_{t} \\
\eta(z)=\mathbf{v}_{t}^{H} \mathbf{T}\left(x(z) \mathbf{T}^{H} \mathbf{R T}-z \mathbf{I}\right)^{-1} \mathbf{T}^{H} \mathbf{v}_{t}
\end{array} .\right.
$$

After several calculation steps, it can be shown that the numerator is equal to $\left.\frac{d}{d z} h(z)\right|_{z=0}$. Applying Theorem 1 again, we know $|h(z)-\eta(z)| \rightarrow 0$, so we can approximate the derivative of $h(z)$ by using $\frac{d}{d z} \eta(z)$. After some simplifications, we get

$$
\frac{d}{d z} \eta(z)=\frac{1-x^{\prime}(z)}{(x(z)-z)^{2}} \mathbf{v}_{t}^{H} \mathbf{F}\left(\mathbf{F}^{H} \mathbf{R F}\right)^{-1} \mathbf{F}^{H} \mathbf{v}_{t},
$$

so the ratio is

$$
\frac{\sigma^{2} \xi_{t}}{1-x^{\prime}(0)} \mathbf{v}_{t}^{H} \mathbf{F}\left(\mathbf{F}^{H} \mathbf{R F}\right)^{-1} \mathbf{F}^{H} \mathbf{v}_{t}
$$

which gives the SINR scaling law as a function of the secondary data support.

\section{Simulations}

Some simulations are included here to verify the theoretical results obtained in the previous sections. The simulated system is equipped with a planar array, and a constant gamma model is considered for the clutter patches [1]. The number of transmitted pulses is 4 for Fig. 3 and 4, and 8 for Fig. 5. In Fig. 3, the antenna configuration is $3 \times Q$, where $Q$ grows from 14 to 110 . In Fig. 4, a $3 \times 110$ rectangular array is used, while in Fig. 5 it is $2 \times 73$. The other simulation parameters can be found in Table I.

Fig. 3 shows the change in SINR as a function of the number of total antenna elements. It can be observed that the performance of the fully adaptive STAP algorithm is always very close to the theoretical upper bound, while the performance of the reduced-dimension separable STAP algorithm is rather poor when the number of antenna elements is small, but it 


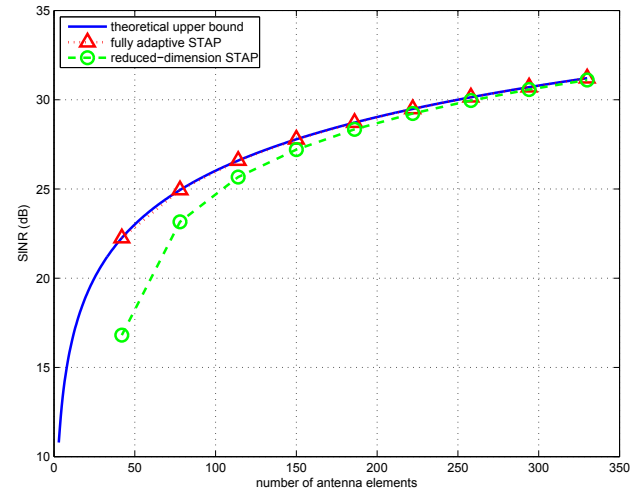

Fig. 3: Max. SINR as a function of the number of antenna elements.

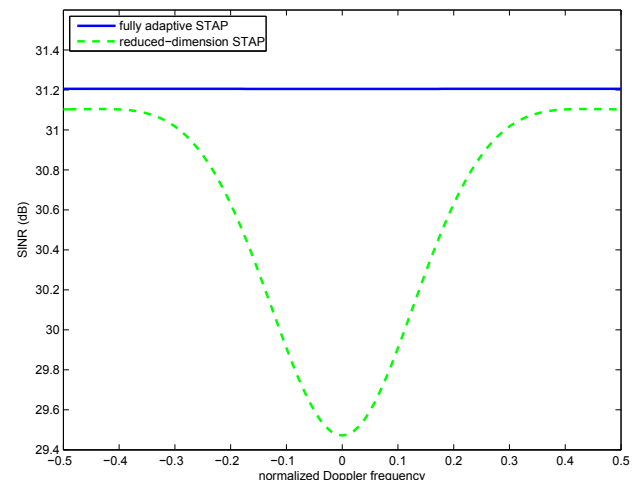

Fig. 4: SINR as a function of normalized doppler frequency.

improves and eventually approaches the fully adaptive STAP algorithm as $N$ grows large. Fig. 4 shows the SINR change as a function of the target speed for a fixed number of antenna elements. When the target is relatively stationary, the fully adaptive algorithm exhibits almost no SINR loss, while the loss of the separable algorithm is less than $2 \mathrm{~dB}$.

Fig. 5 shows two SINR curves; one of them is obtained by using SMI and estimating the interference and noise covariance matrix $\mathbf{R}$, while the other corresponds to the scaling law of (20) predicted by Theorem 1. Although the simulated curve fluctuates, it matches the theoretical curve well when then number of samples is large enough.

\section{CONCLUSION}

In this paper, we have analyzed the performance of a reduced-dimension separable STAP algorithm under the assumption of a very large number of antennas. We showed that the performance loss incurred by the separable algorithm vanishes as the number of antenna elements grow. We also analyzed the performance of the algorithm when it is implemented with a sample covariance estimate, and we found a scaling law that accurately mirrors performance as a function of the number antennas.

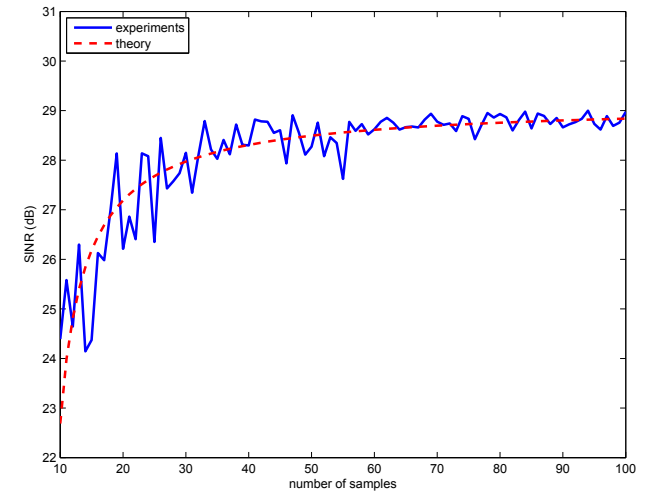

Fig. 5: SINR as a function of the number of samples.

\section{REFERENCES}

[1] J. Ward, "Space-time adaptive processing for airborne radar," Technical Report 1015, MIT Lincoln Laboratory, Lexington, MA, 1994.

[2] W. L. Melvin, "A STAP overview," IEEE Aero. El. Sys. Mag., vol. 19, no. 1 , pp. 19-35, 2004.

[3] C. D. Peckham, A. M. Haimovich, T. F. Ayoub, J. S. Goldstein, and I. S. Reid, "Reduced-rank STAP performance analysis," IEEE Trans. Aerosp. Electron. Syst., vol. 36, no. 2, pp. 664-676, 2000.

[4] Joseph R. Guerci, Space-time adaptive processing for radar, Artech House, 2003.

[5] A. M. Haimovich and Y. Bar-Ness, "An eigenanalysis interference canceler," IEEE Trans. Signal Process., vol. 39, no. 1, pp. 76-84, 1991.

[6] A. Haimovich, "The eigencanceler: adaptive radar by eigenanalysis methods," IEEE Trans. Aerosp. Electron. Syst., vol. 32, no. 2, pp. 532 542, 1996.

[7] D. A. Pados and G. N. Karystinos, "An iterative algorithm for the computation of the MVDR filter," IEEE Trans. Signal Process., vol. 49, no. 2, pp. 290-300, 2001.

[8] J. Scott Goldstein, Irving S. Reed, and L. L. Scharf, "A multistage representation of the Wiener filter based on orthogonal projections," IEEE Trans. Inf. Theory, vol. 44, no. 7, pp. 2943-2959, 1998.

[9] M. L. Honig and J. Scott Goldstein, "Adaptive reduced-rank interference suppression based on the multistage Wiener filter," IEEE Trans. Commun., vol. 50, no. 6, pp. 986-994, 2002.

[10] P. Parker and A. L. Swindlehurst, "Space-time autoregressive filtering for matched subspace STAP," IEEE Trans. Aerosp. Electron. Syst., vol. 39, no. 2, pp. 510-520, 2003.

[11] Romain Couillet and Merouane Debbah, Random matrix methods for wireless communications, Cambridge University Press, 2011.

[12] X. Mestre and M. A. Lagunas, "Finite sample size effect on minimum variance beamformers: optimum diagonal loading factor for large arrays," IEEE Trans. Signal Process., vol. 54, no. 1, pp. 69-82, 2006.

[13] Bo Tang, Jun Tang, and Yingning Peng, "Clutter nulling performance of SMI in amplitude heterogeneous clutter environments," IEEE Trans. Aerosp. Electron. Syst., vol. 49, no. 2, pp. 1366-1373, 2013.

[14] B. Tang, J. Tang, and Y. Peng, "Performance of knowledge aided space time adaptive processing," IET Radar, Sonar \& Navigation, vol. 5, no. 3, pp. 331, 2011

[15] J. Ward, "Space-time adaptive processing for airborne radar," in International Conference on Acoustics, Speech, and Signal Processing, 1995, vol. 5, pp. 2809-2812.

[16] Todd B. Hale, Airborne radar interference suppression using adaptive three-dimensional techniques, Ph.D. thesis, Air Force Institute of Technology, 2002.

[17] Xavier Mestre, "On the asymptotic behavior of quadratic forms of the resolvent of certain covariance-type matrices," Tech. Rep. CTTC/RC/2006-01, Centre Tecnològic de Telecomunicacions de Catalunya, 2006.

[18] Jie Chen, Feng Jiang, and A. Lee Swindlehurst, "On the performance of a separable STAP algorithm for massive antenna arrays," submitted to IEEE Trans. Aerosp. Electron. Syst. 